\title{
Successful catheter ablation of atrial fibrillation from superior venous approach.
}

\author{
Vincenzo Gionti ${ }^{1}$, Massimo Longobardi ${ }^{1}$, Maria Negro ${ }^{2}$, Palma Tartaglione ${ }^{3}$, Emanuela \\ Broglia $^{1}$, Elena Cannas ${ }^{4}$, and Cesare Storti ${ }^{1}$ \\ ${ }^{1}$ Istituto di Cura Città di Pavia \\ ${ }^{2}$ Istituto Di Cura Citta Di Pavia \\ ${ }^{3}$ Prokardia \\ ${ }^{4}$ Istituto di Cura Citta di Pavia
}

November 12, 2020

\begin{abstract}
Background: Trans-catheter ablation of drug refractory atrial fibrillation (AF) is an effective and safe treatment to avoid arrhythmia recurrences. However, some patients may present anatomic variant making impossible an inferior venous approach. We report a case of pulmonary veins isolation (PVI) with cryoballoon technology via right internal jugular vein (RIJV) access. Methods: After the first trans catheter PVI attempt was failed due to inferior vena cava (IVC) hypoplasia, cryoballoon ablation with a 28-mm cryoballoon (AFAPRO - Medtronic CryoCath LP, Quebec, Canada) via superior venous access was performed. A quadripolar electrode was inserted in coronary sinus from left basilic vein. The right internal jugular vein was accessed with Seldinger technique and left atrial (LA) catheterization was performed via single trans-septal puncture (TP) under transesophageal echocardiographic (TOE) and fluoroscopy guidance using an SL2 (St. Jude Medical) trans-septal sheath with a BRK2 (St. Jude Medical) needle. All pulmonary veins (PVs) were engaged. Cryothermal energy was delivered after checking the correct occlusion and PVI was successfully obtained for all PVs. The procedure lasted 210 min, fluoroscopy time was 55 min. During the 16-month follow-up no sustained atrial arrhythmia recurrence was detected. Conclusion: PVI with cryoballoon technology in patients with hypoplasia of IVC can be safely and successfully performed using right internal jugular access.
\end{abstract}

\section{Successful catheter ablation of atrial fibrillation from superior venous approach.}

Gionti Vincenzo ${ }^{1}$, Longobardi Massimo ${ }^{1}$, Negro Maria Claudia ${ }^{1}$, Tartaglione Palma ${ }^{2}$, Broglia Emanuela ${ }^{1}$, Cannas Elena ${ }^{1}$, Storti Cesare ${ }^{1}$.

1 Department of Cardiology, Istituto di Cura Città di Pavia, 27100 Pavia

2 Prokardia SRL, 27100 Pavia.

Corresponding author: Gionti Vincenzo, e-mail giontiv.md@gmail.com

Funding: none.

Disclosure Statement: none.

\section{Background:}

Trans-catheter ablation of drug refractory atrial fibrillation (AF) is an effective and safe treatment to avoid arrhythmia recurrences. However, some patients may present anatomic variant making impossible an inferior venous approach. We report a case of pulmonary veins isolation (PVI) with cryoballoon technology via right internal jugular vein (RIJV) access. 


\section{Methods:}

After the first trans catheter PVI attempt was failed due to inferior vena cava (IVC) hypoplasia, cryoballoon ablation with a 28-mm cryoballoon (AFAPRO - Medtronic CryoCath LP, Quebec, Canada) via superior venous access was performed. A quadripolar electrode was inserted in coronary sinus from left basilic vein. The right internal jugular vein was accessed with Seldinger technique and left atrial (LA) catheterization was performed via single trans-septal puncture (TP) under trans-esophageal echocardiographic (TOE) and fluoroscopy guidance using an SL2 (St. Jude Medical) trans-septal sheath with a BRK2 (St. Jude Medical) needle. All pulmonary veins (PVs) were engaged. Cryothermal energy was delivered after checking the correct occlusion and PVI was successfully obtained for all PVs. The procedure lasted $210 \mathrm{~min}$, fluoroscopy time was 55 min. During the 16-month follow-up no sustained atrial arrhythmia recurrence was detected.

\section{Conclusion:}

PVI with cryoballoon technology in patients with hypoplasia of IVC can be safely and successfully performed using right internal jugular access.

\section{Introduction}

Catheter ablation has been validated as treatment for refractory atrial fibrillation $(\mathrm{AF})$ and its use has increased exponentially in the clinical field ${ }^{1}$. The standard procedure is performed via the right femoral vein, and all devices are designed to be delivered via the femoral venous approach. However, in patients with anatomical obstruction of IVC, i.e. IVC hypoplasia, an alternative technique and vascular access are required. It has been reported that trans-jugular vein access is feasible for catheter ablation of AF, with both technology (cryoballoon and radiofrequency) ${ }^{2-6}$. We report successful cryoablation of paroxysmal AF via RIJV in a patient with hypoplasia of IVC. We describe our technique to perform the TP and to accomplish cryoballoon ablation from superior venous access.

\section{Case Report}

A 54-year-old male with a history of hypertension was admitted to the hospital with a diagnosis of paroxysmal $\mathrm{AF}$ for over 4 years. The paroxysms of AF were becoming more frequent despite treatment with Class $1 \mathrm{C}$ antiarrhythmic drugs in combination with B-blockers. Echocardiography showed no underlying structural heart disease with the LA antero-posterior diameter of $3.9 \mathrm{~cm}$. After considering potential risks and benefits, cryoballoon ablation for treatment of the AF was strongly suggested. After written informed consent was obtained, we planned an electrophysiological study and cryoablation procedure via the right femoral vein. However, the procedure could not be performed because the guidewire did not pass upward through the IVC. Venography revealed a completely hypoplasia of IVC (figure 1) that drained into the superior vena cava via the azygos vein (figure 1). At this stage, we decided to perform LA ablation in another session through the RIJV.

\section{Transseptal puncture from the jugular vein}

Access to the RIJV was obtained with the Seldinger technique using a short 7-F sheath. The short sheath was replaced with an 8-F SL2 (St. Jude Medical) transseptal sheath. The TP was conducted under TOE and fluoroscopy guidance with a BRK2 (St. Jude Medical) needle. After TP a 16-F Flexcath (Medtronic CryoCath LP, Quebec, Canada) was introduced in LA on the guidewire. The TOE probe was, subsequently, removed to prevent a possible atrio-esophageal fistula. Subsequently Heparin was administered intravenously, and an activated clotting time of $300-350 \mathrm{~s}$ was obtained.

\section{Cryoablation procedure}

Cryoablation was started in the left superior pulmonary vein. This vein was engaged by increasing the Flexcath angle to around $120^{*}$. A $28 \mathrm{~mm}$ second-generation cryoballoon catheter (AFAPRO, Medtronic) was positioned in the vein over the octapolar inner-lumen mapping catheter (Achieve VR, Medtronic). After checking the complete occlusion, cryotermal energy was delivered for 240 seconds (temperature nadir $45^{*} \mathrm{C}$ ), with a time to isolation (TTI) of $41 \mathrm{sec}$ (Figure 2A). Subsequently, with a counterclockwise rotation 
of the Flexcath, a mapping catheter was positioned in the right superior pulmonary vein (Figure 2B). The cryoballoon was inflated and complete occlusion of the pulmonary vein was confirmed. A single 240-second application of cryothermal energy was delivered with a temperature nadir of $-55^{*} \mathrm{C}$ and a TTI of 38 sec. The right inferior pulmonary vein was engaged by decreasing the Flexcath angle. A single 240-second application of cryothermal energy was delivered with a temperature nadir of $-49^{*} \mathrm{C}$ (Figure $2 \mathrm{C}$ ) and a TTI of 43 sec. In the 90th second of cryoablation, the balloon was slightly pushed forward to further improve the tissue contact in the inferior portion of the vein. To prevent injury to the phrenic nerve, the quadripolar catheter was withdrawn from his bundle and cryoablations of the right veins were performed under continuous phrenic stimulation. Finally, the left inferior pulmonary vein was engaged in changing the balloon size to $24 \mathrm{~mm}$ (Figure 2D) because the steric hindrance of the $28 \mathrm{~mm}$ balloon due to the relationship between the TP and left inferior PV ostium on the frontal plane. At the end of the procedure, all PVs were checked with the Achieve mapping catheter. Bidirectional blocks were demonstrated for all PVs. A post procedural echocardiography control revealed no pericardial effusion. The total procedure and fluoroscopy times were 210 minutes and 81 minutes, respectively. The patient was discharged from the hospital on the third day after.

\section{Follow Up}

Follow-up was performed according to our usual planning at 3,6 and 12 months as well as in case of symptoms suggestive of arrhythmia recurrence and included a $24 \mathrm{~h}$-Holter recording. After blanking period (BP) the Class 1C antiarrhythmic drug was withdrawn. Any documented sustained episode of AF and/or atrial tachycardia (AT) and/or atrial flutter (AFL) after BP were considered as a recurrent arrhythmic event. After 16 months of follow-up only a self-terminated no sustained episode of AFL was recorded in BP.

\section{Discussion}

Hypoplasia of the IVC is uncommon ${ }^{7}$ and it is usually associated with direct hepatic venous drainage into the RA and venous drainage of the lower body into the azygous system at the level of the renal veins. ${ }^{8}$ For management of drug refractory symptomatic AF in patients with complete interruption of the IVC, several treatment strategies, such as His bundle ablation and pacemaker implantation or surgical AF ablation, could be considered. Furthermore, on the basis of our experience, AF catheter ablation via the superior venous approach may be considered as a therapeutic option when a femoral venous approach is not available.

In cases of the superior approach, due to different distance between SVC-RA and fossa ovalis an angulated sheath, such as SL2 SJM is needed. Also, a steerable sheath may make transseptal puncture a little easier, although we have not used it in our patients. In cases of difficult transseptal puncture, transseptal puncture guided by TOE can be easily and safely performed with the identification of all adjacent structures, which helps prevent major complications such as aortic or atrial perforation or laceration. Alternatively, intracardiac echocardiography (ICE) would provide important anatomical details of the interatrial septum and greater certainty of a safe puncture site and of introducing the long sheath into the LA. However, in cases of the superior approach, the use of ICE is limited because it requires a separate vascular access. In the present cases the use of TOE is sufficient to support the optimal position of the needle tip and to enable us to perform a safe TP. After successful TP, we easily introduce the steerable sheath into the LA using a stiff guide-wire. There are several descriptions of TP with superior venous approach and all the cases described a more angulated needle and sheath are needed. The electrical isolation was achieved for all the PVs. The principal difficulty was encountered to achieve a complete occlusion of left inferior PV because the relationship between the TP and left inferior PV ostium on the frontal plane. We reached it changing balloon size from $28 \mathrm{~mm}$ to $24 \mathrm{~mm}$. The smallest balloon diameter, in our case, leads to easier occlusion of the left inferior PV ostium.

\section{Conclusion}

We described the safety and feasibility of balloon cryoablation for AF via RIJV in a patient with an interrupted IVC.

\section{References}


1) Cappato R, Calkins H, Chen SA, Davies W, Iesaka Y, Kalman J, Kim YH, Klein G, Packer D, Skanes A. Worldwide survey on the methods, efficacy, and safety of catheter ablation for human atrial fibrillation. Circulation. 2005;111:1100-1105.

2) Kanat S, Cakir H, Tutuncu A, et al. Successful cryoablation of atrial fibrillation from jugular approach in patient with interrupted inferior vena cava and azygos continuation, Pacing Clin Electrophysiol. 2019;42:309312.

3) Lim HE, Pak HN, Tse HF, et al Catheter ablation of atrial fibrillation via superior approach in patients with interruption of the inferior vena cava. Heart Rhythm 2009;6:174-179

4) Baszko A, Kałmucki P, Dankowski R, et al. Transseptal puncture fromthe jugular vein and balloon cryoablation for atrial fibrillation in a patient with azygos continuation of an interrupted inferior vena cava. Europace (2015) 17, 1153-1156

5) Kato H, Kubota S, Goto T, et al. Transseptal puncture and catheter ablation via the superior vena cava approach for persistent atrial fibrillation in a patient with polysplenia syndrome and interruption of the inferior vena cava: contact force-guided pulmonary vein isolation. Europace (2017) 19, 1227-1232

6) Masaki K, Morishige K, Matsusaka H, Kubo T. Radiofrequency catheter ablation of atrial fibrillation through an implanted inferior vena cava filter. J Cardiol Cases. 2019;19(5):161-164.

7) Blanchard DG, Sobel JL, Hope J, et al. Infrahepatic interruption of the inferior vena cava with azygos continuation: a potential mimicker of aortic pathology. J Am Soc Echocardiogr 1998;11:1078 -1083.

8) Koc Z, Oguzkurt L. Interruption or congenital stenosis of the inferior vena cava: prevalence, imaging, and clinical findings. Eur J Radiol 2007;62:257-266.

Figure 2

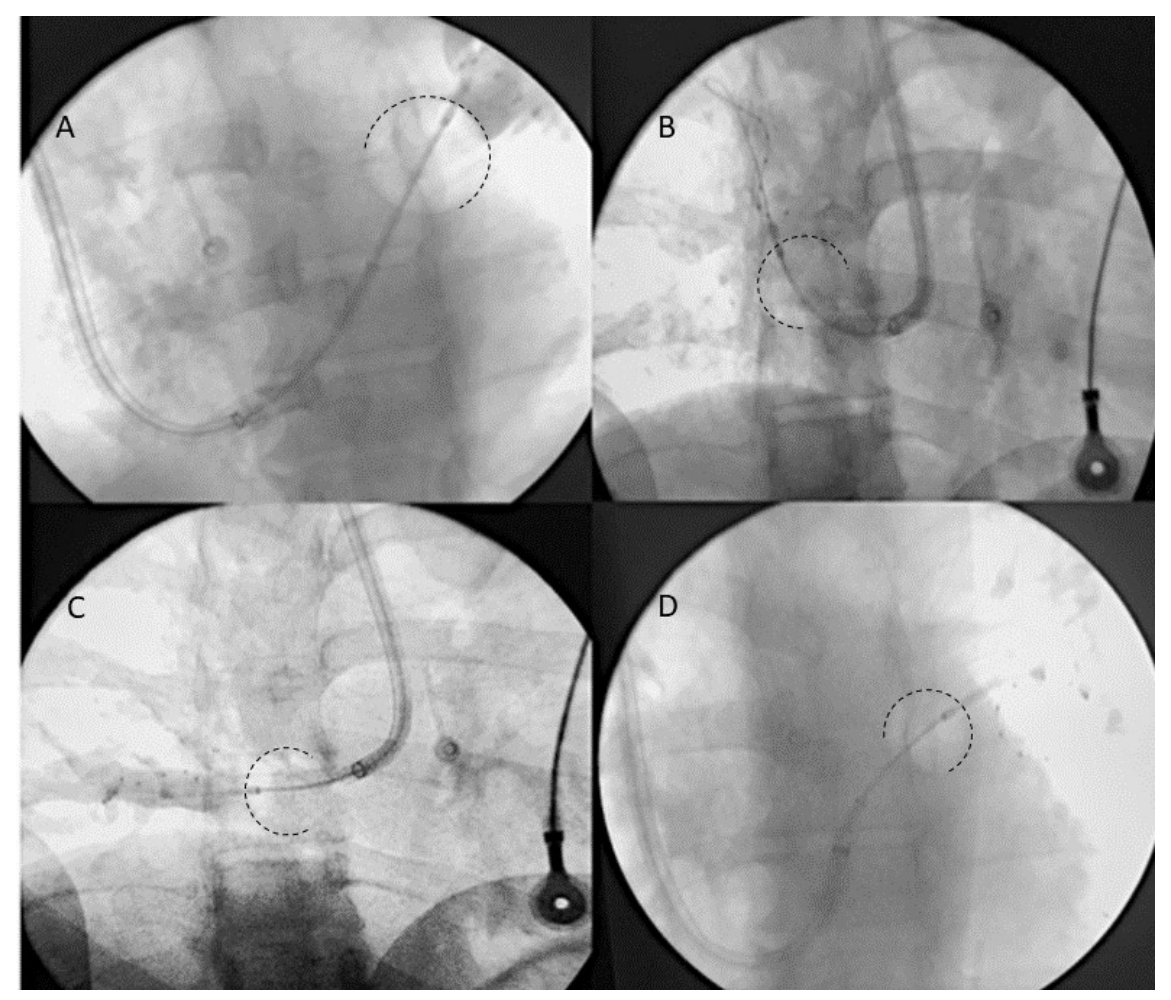


The images show the fluroscopic views of the balloon during cryoablation of the veins: (A) left superior PV; (B) right superior PV; (C) right inferior PV; (D) left inferior PV.

Fig 2

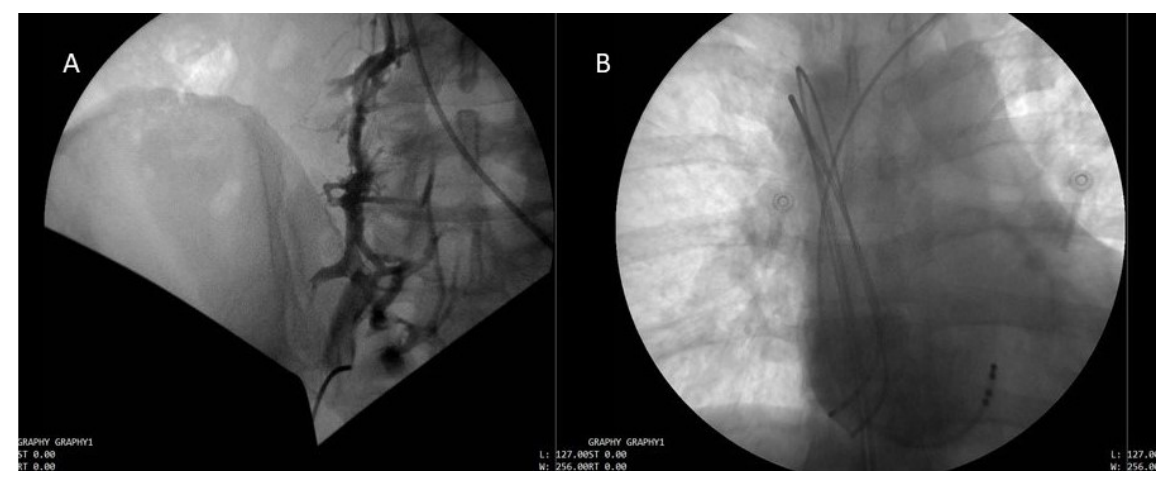

The images show the hypolasia of IVC (A) and the access to right atrium via azygos vein (B). 